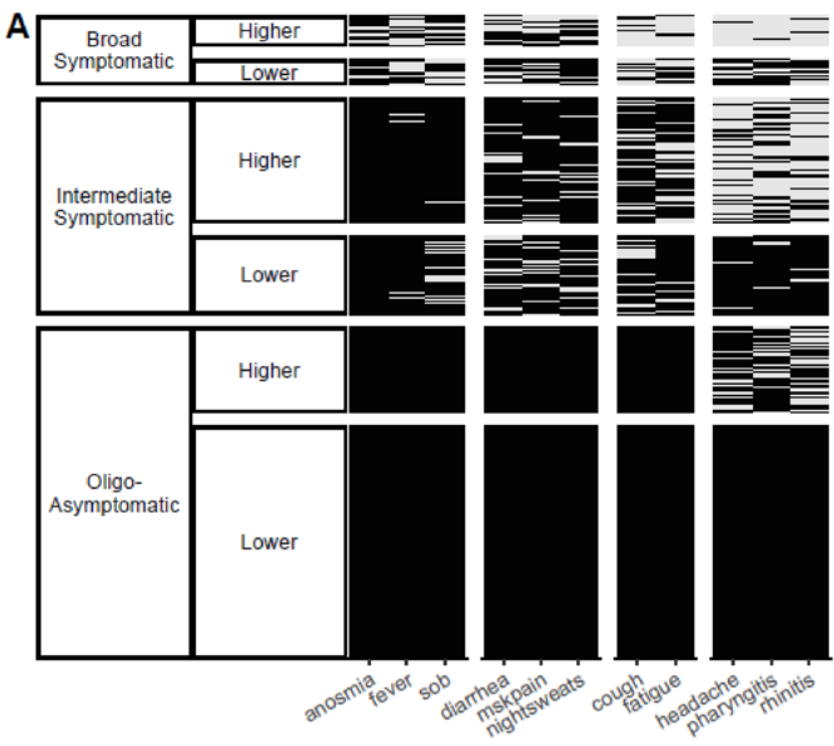

B

Broad Symptomatic
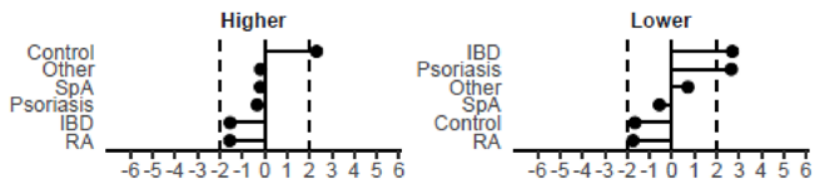

Intermediate Symptomatic
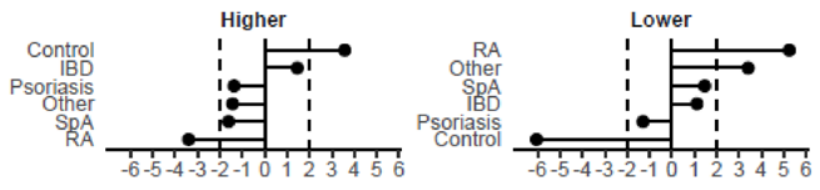

Oligo-/Asymptomatic

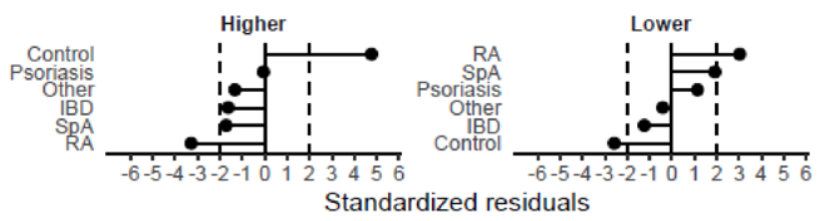

Figure 1. A) distribution of common viral respiratory disease symptoms across patient and symptom clusters. B) Standardized residuals indicating deviation from expected frequencies of IMID diagnoses across patient clusters. sob: shortness of breath, mskpain: musculoskeletal pain

Conclusion: This analysis shows that symptoms of common respiratory viral infections are less frequent in RA patients and to a lesser extent in other IMID patient. As major clusters in this analysis can also be considered to represent exposure categories, these data suggest that IMIDs or their treatments may mitigate common respiratory viral infection symptoms.

REFERENCES:

[1] Simon D. et al. Nat Commun (2020) 11, 3774

[2] Bhatia P. et al. J. Stat. Softw (2017) 76(9)

Acknowledgements: This study was supported by the Deutsche Forschungsgemeinschaft (DFG- FOR2886 PANDORA and the CRC1181), the Bundesministerium für Bildung und Forschung (BMBF; project MASCARA), the H2020 GA 810316 - 4D-Nanoscope ERC Synergy Project, the IMI funded project RTCure, the Emerging Fields Initiative MIRACLE of the Friedrich-AlexanderUniversität Erlangen-Nürnberg as well as the Schreiber Stiftung gemeinnützige Gesellschaft $\mathrm{mbH}$.

Disclosure of Interests: None declared

DOI: 10.1136/annrheumdis-2021-eular.1598

\section{POS1427 NATIONAL BURDEN OF RHEUMATOID ARTHRITIS IN CANADA 1990-2017: FINDINGS FROM THE GLOBAL BURDEN OF DISEASE STUDY 2017}

N. Hassen ${ }^{1}$, D. Lacaille ${ }^{1}$, A. Xu ${ }^{1}$, S. Sidi ${ }^{1}$, A. Alandejani ${ }^{1}$, M. Mansourian ${ }^{2}$, N. Sarrazadegan ${ }^{1}$, J. Kopec ${ }^{1} .{ }^{1}$ The University of British Columbia, Faculty of Medicine, Vancouver, Canada; ${ }^{2}$ Isfahan University of Medical Sciences, Isfahan, Iran (Islamic Republic of)

Background: According to the Global Burden of Diseases, Injuries, and Risk Factors Study (GBD) 2017, over 120,000 individuals currently have rheumatoid arthritis (RA) in Canada, yet a study that evaluates the combined effect of RA on the longevity and quality of life in the country is lacking.

Objectives: The objectives of this study are three: 1) to describe burden of RA levels and trends from 1990-2017 using GBD data, 2) to describe age and sex differences, and 3) to compare Canada RA burden to other countries.

Methods: We obtained publicly available data from GBD Study 2017 from the Institute for Health Metrics and Evaluation interactive visualization tool (http:// vizhub.healthdata.org/gbd-compare). Disease burden indicators include prevalence, mortality, years of life lost (YLLs), years lived with disability (YLDs), and disability-adjusted life years (DALYs). Estimates were presented as non age-standardized and age-standardized rates per 100,000 population. GBD estimated prevalence using published literature, survey data, patient records, and health insurance claims, and mortality using cause of death ensemble modelling technique. YLLs measure premature death calculated as the sum of each death multiplied by the standard life expectancy at each age. YLDs measure amount of time in a year an individual lives with a short- or long-term health condition, calculated by combining prevalence with disability weights for each age/sex/year. DALYs were calculated as the sum of YLLs and YLDs. DALYs for Canada were compared to DALYs of countries with similar socio-demographic index (SDI) values. SDI combines income per capita, average educational attainment, and fertility rates. Data were analysed by DisMod-MR 2.1, a Bayesian meta-regression tool.

Results: In Canada, RA mortality (mortality and YLLs) improved over time, with a steeper decline after the year 2002. However, the population burden of quality of life (YLDs and DALYs) increased due to increasing prevalence. The disease burden was higher in females (prevalence, mortality, YLLs, YLDs, DALYs), and DALY rates were higher among older populations. Compared to other countries, Canada had greater improvement in mortality and YLLs over time and had a lower age-standardized DALYs rate compared to countries of similar SDI values. A weak association was found between global age-standardized DALYs and SDI $\left(R^{2}=0.0138\right)$

Conclusion: RA is a major public health challenge. Canada fares better than other countries with regards to national RA burden. Early identification and management are critical to reducing the overall burden of RA in Canada, especially in women. More data from multiple provincial RA databases would increase the accuracy of our estimates for Canada.

\section{REFERENCES:}

[1] Cross M, Smith E, Hoy D, et al. The global burden of rheumatoid arthritis: estimates from the global burden of disease 2010 study. Ann Rheum Dis. 2014;73(7):1316-1322. doi:10.1136/annrheumdis-2013-204627

[2] Myasoedova E, Davis JM 3rd, Crowson CS, Gabriel SE. Epidemiology of rheumatoid arthritis: rheumatoid arthritis and mortality. Curr Rheumatol Rep. 2010;12(5):379-385. doi:10.1007/s11926-010-0117-y

[3] Safiri S, Kolahi AA, Hoy D, et al. Global, regional and national burden of rheumatoid arthritis 1990-2017: a systematic analysis of the Global Burden of Disease study 2017. Ann Rheum Dis. 2019;78(11):1463-1471. doi:10.1136/ annrheumdis-2019-215920

Acknowledgements: Nejat Hassen is supported by a grant from the Canadian Institute of Health Research. Dr. Diane Lacaille is supported by the Mary Pack Chair in Arthritis Research from the University of British Columbia and The Arthritis Society of Canada.

Disclosure of Interests: None declared

DOI: 10.1136/annrheumdis-2021-eular.1927

\section{POS1428 VALIDATION OF ANCA-ASSOCIATED VASCULITIS AS THE CAUSE OF END-STAGE RENAL DISEASE IN THE UNITED STATES RENAL DATA SYSTEM}

C. Cook ${ }^{1}$, H. Choi ${ }^{1,2}$, Z. Wallace ${ }^{1,2} .{ }^{1}$ Massachusetts General Hospital, Division of Rheumatology, Allergy, and Immunology, Boston, United States of America;

${ }^{2}$ Harvard, Medical School, Boston, United States of America

Background: Glomerulonephritis and other renal manifestations are common in ANCA-associated vasculitis (AAV). Renal involvement in AAV is associated with adverse outcomes, including end-stage renal disease (ESRD) in up to $25 \%$ of patients (1). The United States Renal Data System (USRDS), a national registry of ESRD patients, represents a unique nationwide data source for studying AAV patients with ESRD. Prior research has assessed how often patients with ESRD attributed to AAV have biopsy-proven glomerulonephritis in USRDS (2), but the validity of the diagnosis of AAV as the cause of ESRD in the USRDS remains unknown.

Objectives: We aim to validate the diagnosis of AAV as the primary cause of ESRD listed in USRDS. 
Methods: We identified all patients in the Mass General Brigham (MGB) healthcare system with a billing code for advanced chronic kidney disease or end-stage renal disease or procedure code for dialysis or renal transplantation. We identified all MGB patients fulfilling these criteria to records in the USRDS by name, sex, date of birth, and social security number. From this cohort of patients, we identified those with AAV or related diagnoses listed as the primary disease causing ESRD (ICD9: 446.0, 446.4 or ICD10: M31.3X, M31.7). Two authors reviewed medical records to collect information on whether or not a physician had diagnosed AAV, details of AAV history, renal and non-renal biopsies, and antineutrophil cytoplasmic antibody (ANCA) tests. Discrepancies were resolved through consensus. Details regarding initial ESRD onset date were obtained from the USRDS. To calculate the positive predictive value (PPV) for AAV as the primary cause of ESRD a definite physician diagnosis of AAV (a diagnosis confirmed by two physicians based on available data) in the MGB medical record was used as the gold standard. To calculate sensitivity, we linked the Partners (MGB) AAV Cohort to USRDS records using the same methods. A diagnosis code of AAV as the cause of ESRD was considered a true positive and a diagnosis code for other types of nephritis was considered a false negative.

Results: We identified 89 USRDS records linked to MGB medical records in which the primary cause of ESRD was attributed to AAV. Of these, 85 were confirmed to be true cases of AAV after medical record review (PPV=96\%) (Table 1). Among the cases classified as AAV, 84 (99\%) had a positive ANCA test, which was predominantly MPO/P-ANCA (47, 55\%); $36(42 \%)$ had a renal biopsy, all of which were supportive of the diagnosis. The majority of cases were identified as AAV by ICD9 or 10 codes for Wegener's granulomatosis (446.4 or M313.1). Within the Partners (MGB) AAV cohort linked to USRDS records, $33(55 \%)$ of 60 identified cases had AAV listed as the cause of ESRD; in the remainder, ESRD was attributed to non-specific nephritis codes.

Table 1. AAV and non-AAV patients in the USRDS with ESRD due to AAV $(\mathrm{N}=89)$

\begin{tabular}{lc}
\hline & $\begin{array}{c}\text { Physician-Diagnosed AAV } \\
(\mathrm{N}=85)\end{array}$ \\
\hline ANCA type $\mathrm{n}(\%)$ & $84(98.8)$ \\
MPO/P-ANCA+ & $47(55.3)$ \\
PR3/C-ANCA+ & $33(38.8)$ \\
Renal biopsy $n(\%)$ & $36(42.4)$ \\
Pauci-Immune Glomerulonephritis n (\%) & $16(44 \%)$ \\
Non-renal biopsy n (\%) & $10(11.8)$ \\
Yes & $74(87.1)$ \\
No & $1[0,6]$ \\
Years from AAV diagnosis to ESRD median [IQR] & $81(95.3)$ \\
Principal diagnosis code (ICD9/ICD10) $n$ (\%) & \\
$\quad$ Wegener's granulomatosis (446.4, 446.4B, or M313.1) & \\
\hline
\end{tabular}

Conclusion: We found that the diagnosis of AAV as the primary cause of ESRD in the USRD had a high PPV, suggesting accurate classification of ESRD due to AAV in the USRDS, but that sensitivity was moderate. These findings support the past and future use of the USRDS for research with ESRD attributed to AAV. REFERENCES:

[1] Moiseev S, Novikov P, Jayne D, Mukhin N. End-stage renal disease in ANCA-associated vasculitis. Nephrol Dial Transplant. 2017;32(2):248-53.

[2] Layton JB, Hogan SL, Jennette CE, Kenderes B, Krisher J, Jennette JC, et al. Discrepancy between Medical Evidence Form 2728 and renal biopsy for glomerular diseases. Clin J Am Soc Nephrol. 2010;5(11):2046-52.

Disclosure of Interests: None declared

DOI: 10.1136/annrheumdis-2021-eular.2138

\section{POS1429 ORAL DYSBIOSIS REFLECTS THE IMMUNOLOGICAL ALTERATION OF RA REGARDING TO HLA DRB1*SE, ACPA AND CIGARETTE SMOKING: NAGASAKI ISLAND STUDY}

Y. Tsuji ${ }^{1}$, M. Tamai ${ }^{2}$, S. Morimoto ${ }^{3}$, D. Sasaki ${ }^{2}$, S. Y. Kawashiri' ${ }^{1}$ K. Yanagihara ${ }^{2}$, K. Aoyagi ${ }^{4}$, T. Maeda ${ }^{1}$, F. Matsuda ${ }^{5}$, A. Kawakami ${ }^{4}$, T. Saito $6 .{ }^{1}$ Nagasaki University, Department of Community Medicine, Graduate School of Biomedical Sciences, Nagasaki, Japan; ${ }^{2}$ Nagasaki University, Department of Laboratory Medicine, Nagasaki, Japan; ${ }^{3}$ Nagasaki University, Department of Public Health, Graduate School of Biomedical Sciences, Nagasaki, Japan; ${ }^{4}$ Nagasaki University, Department of Immunology and Rheumatology, Nagasaki, Japan; ${ }^{5}$ Kyoto University, Center for Genomic Medicine, Kyoto, Japan;

${ }^{6}$ Nagasaki University, Department of Oral Health, Medical and Dental Sciences, Nagasaki, Japan
Background: Anti-citrullinated protein antibody (ACPA) production is observed in several organs even prior to the onset of rheumatoid arthritis (RA), and oral mucosa is considered to be one of the important tissues. Saliva is considered to reflect the oral microbiota(oralMB) including periodontal disease. A gene-environment interaction between cigarette smoking and shared epitope genes in HLA-DRB1* shared epitope (SE) provides a high risk of ACPA-positive RA. However, the interaction of $\mathrm{HLA}-\mathrm{DRB} 1{ }^{*} \mathrm{SE}, \mathrm{ACPA}$, cigarette smoking and oralMB of RA patients remains to be elucidated.

Objectives: We investigated that the difference of oralMB among RA patients and healthy subjects(HS) regarding to ACPA, HLA-DRB1*SE and cigarette smoking.

Methods: The Nagasaki Island Study, which had started in 2014 collaborating with Goto City, Nagasaki Prefecture, Japan, is intended for research of the preclinical stage of RA, including ACPA, HLA genotype screening, oralMB and lifestyle habit. Both of blood and salivary samples were obtained from 1422 subjects out of 4276 participants in this study from 2016 to 2018 . ACPA positivity was $1.7 \%$ in total 4276 subjects. At this point, we selected 291 subjects, who were ACPA positive non-RA HS $(n=22)$ and patients with RA ( $n=33,11$ subjects were ACPA positive and 22 ACPA negative, respectively) as the case, age and gender matched ACPA negative non-RA HS ( $n=236)$ as the control. In RA subjects, current smoker was $n=1(3.0 \%)$ and ever smoker was $n=8(24.2 \%)$. In HS, current smoker was $n=29(11.2 \%)$ and ever smoker was $n=55(21.3 \%)$. ACPA was measured by ELISA, and HLA genotyping was quantified by next-generation sequencing (Ref.1). The operational taxonomic unit (OTU) analysis using 16S rRNA gene sequencing were performed. The richness of microbial diversity within subject ( $\alpha$-diversity) was scaled via Shannon entropy. The dissimilarity between microbial community composition was calculated using Bray-Curtis distance as a scale, and differences between groups ( $\beta$-diversity) were tested by permutational multivariate analysis of variance (PERMANOVA). In addition, UniFrac distance calculated in consideration of the distance on the phylogenetic tree were performed.

Results: Median age 71 y.o., \% Female $58.4 \%$. Among RA and non-RA subjects, not $\alpha$-diversity but $\beta$-diversity was statistically smaller significantly in RA $(p=0.022)$. In the HS, there was no decrease in $\alpha$-diversity between the ACPA-positive and HLA-DRB1*SE-positive groups, but in the ACPA-positive group, there was a decrease in $\alpha$-diversity in the HLA-DRB1*SE-positive group. When we compared $\alpha$-diversity stratified by the presence or absence of three factors (RA, ACPA, and HLA-DRB1*SE), the RA group with ACPA and $\mathrm{HLA}-\mathrm{DRB} 1{ }^{*} \mathrm{SE}$ positive tended to have the lowest diversity (Figure 1 lower right). RA subjects, presence of HLA$\mathrm{DRB} 1{ }^{\star} \mathrm{SE}$ did not show the difference but the tendency of lower $\alpha$-diversity $(p=0.29)$.

Conclusion: HS with ACPA-positive HLA-DRB1*SE tended to show lower $\alpha$-diversity than ACPA-positive HS and HLA-DRB1*SE positive HS. Furthermore, RA subjects with ACPA-positive HLA-DRB1 ${ }^{*}$ SE showed lower $\alpha$-diversity than HS with ACPA-positive HLA-DRB1*SE.

Our study suggested that the oral dysbiosis may reflect the immunological status of patients with RA. Because of the small number of ACPA-positive patients, stratification by smoking history was difficult. Further examination is needed to clarify the gene-environment interaction and microbiome.

REFERENCES:

[1] Kawaguchi S, et al. Methods Mol Biol 2018;1802: 22.

\section{Comparison of $\alpha$ diversity by ACPA positivity, HLA -DRB1 possession, and RA}

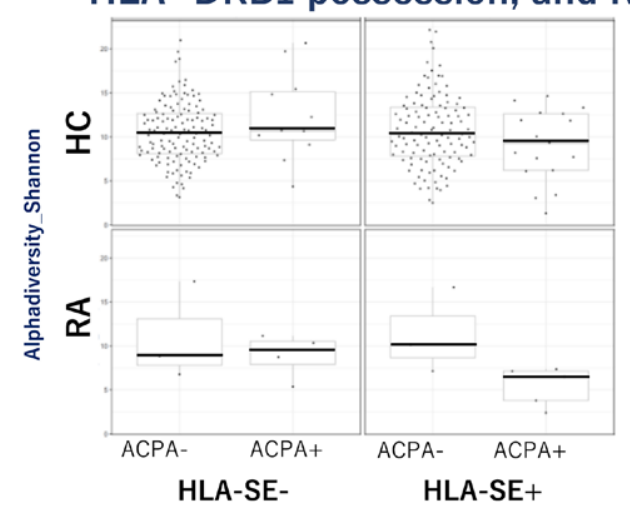

Disclosure of Interests: None declared

DOI: 10.1136/annrheumdis-2021-eular.2270 